\title{
Stationary discrete solitons in a driven dissipative Bose-Hubbard chain
}

\author{
Uta Naether, ${ }^{1, *}$ Fernando Quijandría, ${ }^{1}$ Juan José García-Ripoll, ${ }^{2}$ and David Zueco ${ }^{1,3}$ \\ ${ }^{1}$ Instituto de Ciencia de Materiales de Aragón y Departamento de Física de la Materia Condensada, CSIC-Universidad de Zaragoza, \\ Zaragoza E-50012, Spain \\ ${ }^{2}$ Instituto de Física Fundamental, IFF-CSIC, Serrano 113-bis, Madrid E-28006, Spain \\ ${ }^{3}$ Fundación ARAID, Paseo María Agustín 36, Zaragoza E-50004, Spain
}

(Received 1 December 2014; published 18 March 2015)

\begin{abstract}
We demonstrate that stationary localized solutions (discrete solitons) exist in one-dimensional Bose-Hubbard lattices with gain and loss in a semiclassical regime. Stationary solutions, by definition, are robust and do not demand state preparation. Losses, unavoidable in experiments, are not a drawback, but a necessary ingredient for these modes to exist. The semiclassical calculations are complemented with their classical limit and dynamics based on a Gutzwiller ansatz. We argue that circuit quantum electrodynamic architectures are ideal platforms for realizing the physics developed here. Finally, within the input-output formalism, we explain how to experimentally access the different phases, including the solitons, of the chain.
\end{abstract}

DOI: 10.1103/PhysRevA.91.033823

PACS number(s): 42.50.Pq, 05.45.Yv, 03.65.Yz, 03.75.Lm

\section{INTRODUCTION}

Realizations of quantum nonlinear media as ultracold atoms in optical lattices [1], ion traps [2] or superconducting circuits $[3,4]$ are interesting candidates for future quantum information processors. Apart from this challenging goal, they are also testbeds to explore new many-body states of matter both in the classical and quantum regime [5]. Among others, discrete solitons-localized and form-preserving solutions of coupled nonlinear lattice equations-are a paradigmatic example of collective nonlinear solutions. In the so-called classical limit of the Bose-Hubbard model, the operators are replaced by their $c$-number average, obtaining the well-known discrete nonlinear Schrödinger equation (DNLS) [6]. In this limit, discrete solitons, both theoretically and experimentally, exist in different dimensions and topologies [7-10].

Quantum solitons have been hypothesized to exist in the Bose-Hubbard model with and without dissipation. Theoretical predictions are based on different approaches. The conservative model was explored with a Gutzwiller ansatz [11] and density matrix renormalization group techniques [12]. The truncated Wigner approximation of [13] considered effective dissipative dynamics, whereas the Gaussian expansions proposed by [14] used explicit damping terms for their dissipative model. All the aforementioned models have found slowly decaying localized solutions. Therefore, none of those were stable solutions for the dynamics. Thus, quantum fluctuations seem to kill these topological solutions. Experimental realizations in the quantum realm are few. Bose-Einstein condensates confirmed the presence of slowly decaying bright [15] and dark [16] localized modes [17]. For ions in optical traps, a proposal [18] was shortly after followed by the experimental observation of long-lived solitons [19].

Things may change if dissipation and gain coexist. In the classical limit yielding the dissipative driven DNLS (DD-DNLS) equation, localized solutions have been reported $[20,21]$. Furthermore, the DD-DNLS exhibits "spontaneous walking" solitons [22]; using nonlinear gain and

\footnotetext{
*naether@unizar.es
}

dissipation, exact traveling discrete solitons exist as stable dynamical attractors [23]. Therefore, an open question remains in the literature: What about quantum solitons in nonlinear media with loss and gain? In our opinion, the combination of many-body physics, dissipation, and driving is interesting. It provides new phases to explore with nonthermal but equilibrated states, as already demonstrated in the dissipative driven Bose-Hubbard model [24,25]. Besides, it establishes a link with manmade realizations of lattice systems where dissipation can be an issue [5]. In the present context these novel phases could provide solitons.

In this work, we discuss the existence of stationary solitons within the dissipative driven Bose-Hubbard. Stationary solitons have an important advantage over exact solutions of conservative equations. Stationary solutions, if stable, are obtained via the dissipative dynamics no matter the initial state (belonging to the basins of attraction). Therefore their preparation is easier and more robust.

In this work, we first argue that for the dissipative driven Bose-Hubbard model quantum solitons have no anticontinuous limit, i.e, the uncoupled lattice system has a unique stationary solution [26-28]. This is important, since the single-site DD-DNLS for the same parameter regime can have different fixed points in their irreversible dynamics. This is a qualitative difference and forces us to be careful in the utilization of the DD-DNLS for finding solitons. As the full problem is intractable analytically, we will include quantum fluctuations up to second order, i.e., beyond the DD-DNLS limit. In doing so, we must guarantee the uniqueness of the stationary single-site solution. Solitons exist within this (we call it) semiclassical approximation. We discuss their stability and range of existence. We also describe other types of phases appearing when the soliton solution is unstable or absent. We complement our study with a Gutzwiller ansatz. We observe that localization is more persistent within the Gutzwiller in the range where solitons exist within the semiclassical limit. Finally, we discuss a physical realization for the dissipative driven Bose-Hubbard based on a circuit quantum electrodynamic (circuit-QED) architecture [29]. The physical support for our model is complemented with a proposal for a measurement scheme based on an input-output theory, 
to access the different phases using (already demonstrated) experimental capabilities.

The rest of the paper is organized as follows. Section II presents the model including dissipation and gain. In addition, we briefly describe the different theoretical approaches used: a second-order (in the quantum fluctuations) expansion (SOE) and the Gutzwiller ansatz. We finish the section with a possible implementation in circuit-QED architectures. In Sec. IV we show our numerical results in both approximation schemes and we compare them against the classical DD-DNLS. We present in Sec. V the input-output formalism for measuring the different phases and conclude with a discussion in Sec. VI.

\section{MODEL AND ITS (APPROXIMATE) SOLUTIONS}

The Bose-Hubbard model with driving reads $(\hbar=1)$

$H=\sum_{l} \delta \omega a_{l}^{\dagger} a_{l}+\frac{U}{2} a_{l}^{\dagger 2} a_{l}^{2}-J\left(a_{l+1}^{\dagger} a_{l}+\right.$ h.c. $)+A\left(a_{l}^{\dagger}+a_{l}\right)$

It marks a minimal model for interacting bosons in a lattice. The model (in the rotating frame of the drive and adimensionalized) is characterized via $\delta \omega$ (the detuning of the bare resonator frequency $\omega_{0}$ from the pump frequency $\omega_{d}, \delta \omega=\left|\omega_{0}-\omega_{d}\right|$ ), $A$ (the driving amplitude for this coherent external driving), $U$ (the onsite repulsion), and $J$ (the strength of the hopping among sites). Phenomenologically, single-particle losses can be cast in a Gorini-Kossakowski-Sudarshan-Lindblad master equation [30],

$$
d_{t} \varrho=-i[H, \varrho]+\gamma \sum_{l} a_{l} \varrho a_{l}^{\dagger}-\frac{1}{2}\left\{a_{l}^{\dagger} a_{l}, \varrho\right\},
$$

with $\gamma^{-1}$ the dimensionless time scale for the losses and $\{A, B\}=A B+B A$ the anticommutator. A pictorial and physical realization based on circuit-QED is shown in Fig. 1.
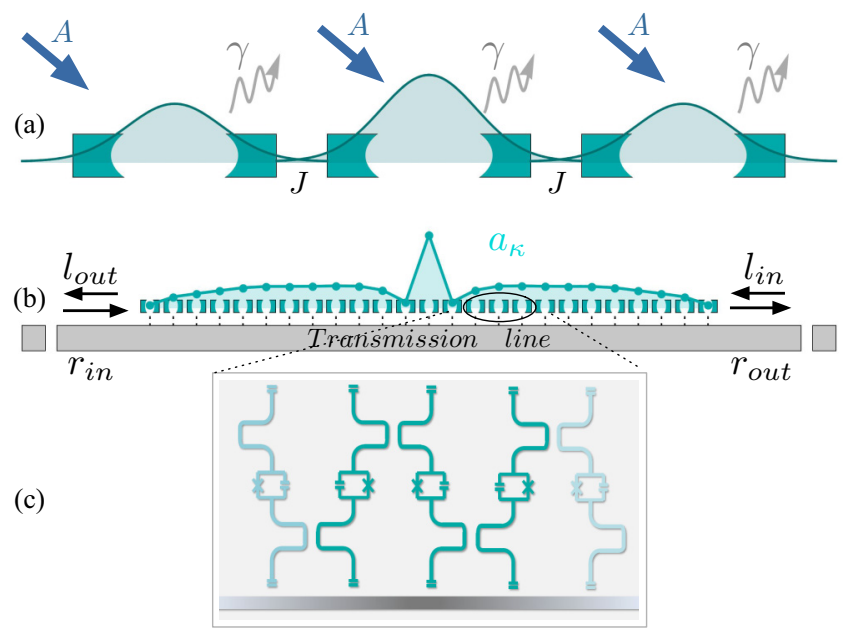

FIG. 1. (Color online) (a) Scheme of the driven and dissipative cavity array and (b) input-output measurement using the interaction of the coupled cavity array with an soliton and a transmission line. (c) Sketch of the circuit proposal; curved lines represent superconducting resonators interrupted by a $\mathrm{JJ}$ (squares with cross and a capacitor.)
Without loss and driving, the Bose-Hubbard is a cornerstone in many-body physics. The generalized Bose-Hubbard Eqs. (1) and (2) mark, then, a paradigmatic model for the study of collective phenomena with driving and dissipation. The dynamical equations for the averages $\left\langle a_{l}^{\dagger} \ldots a_{m}\right\rangle \equiv \operatorname{Tr}\left(a_{l}^{\dagger} \ldots a_{m} \varrho\right)$ are given by

$$
\begin{aligned}
i d_{t}\left\langle a_{l}\right\rangle= & \left(\delta \omega-i \frac{\gamma}{2}\right)\left\langle a_{l}\right\rangle+A-J\left(\left\langle a_{l+1}\right\rangle+\left\langle a_{l-1}\right\rangle\right) \\
& +U\left(2\left\langle a_{l}^{\dagger} a_{l}\right\rangle\left\langle a_{l}\right\rangle+\left\langle a_{l}^{2}\right\rangle\left\langle a_{l}^{\dagger}\right\rangle-2\left\langle a_{l}\right\rangle^{2}\left\langle a_{l}^{\dagger}\right\rangle\right) \\
i d_{t}\left\langle a_{l}^{\dagger} a_{m}\right\rangle= & -i \gamma\left\langle a_{l}^{\dagger} a_{m}\right\rangle+A\left(\left\langle a_{l}^{\dagger}\right\rangle-\left\langle a_{m}\right\rangle\right)+J\left(\left\langle a_{l-1}^{\dagger} a_{m}\right\rangle\right. \\
& \left.+\left\langle a_{l+1}^{\dagger} a_{m}\right\rangle-\left\langle a_{l}^{\dagger} a_{m-1}\right\rangle-\left\langle a_{l}^{\dagger} a_{m+1}\right\rangle\right) \\
& +U\left(\left\langle a_{l}^{\dagger} a_{m}^{\dagger} a_{m} a_{m}\right\rangle-\left\langle a_{l}^{\dagger} a_{l}^{\dagger} a_{l} a_{m}\right\rangle\right) \\
i d_{t}\left\langle a_{l} a_{m}\right\rangle= & (2 \delta \omega-i \gamma)\left\langle a_{l} a_{m}\right\rangle+A\left(\left\langle a_{l}\right\rangle+\left\langle a_{m}\right\rangle\right)-J\left(\left\langle a_{l-1} a_{m}\right\rangle\right. \\
& \left.+\left\langle a_{l+1} a_{m}\right\rangle+\left\langle a_{l} a_{m-1}\right\rangle+\left\langle a_{l} a_{m+1}\right\rangle\right) \\
& +U\left(\left\langle a_{l}^{\dagger} a_{m} a_{m} a_{m}\right\rangle+\left\langle a_{l}^{\dagger} a_{l} a_{l} a_{m}\right\rangle+\delta_{l, m}\left\langle a_{l} a_{l}\right\rangle\right)
\end{aligned}
$$

The dots above indicate that, due to the interaction term $U a_{l}^{\dagger 2} a_{l}^{2}$, an endless hierarchy of equations for the $n$-point correlators $\left\langle a_{l}^{\dagger} \ldots a_{m}\right\rangle$ is obtained. Therefore, the set needs to be cut at some order.

\section{A. Zeroth order: The DD-DNLS equation}

The simplest approximation is the so-called classical limit, consisting of replacing operators by their averages: $\left\langle a_{l}^{\dagger 2} a_{l}\right\rangle \rightarrow\left|\varphi_{l}\right|^{2} \varphi_{l}$, with $\varphi_{l}=\left\langle a_{l}\right\rangle$. The approximation can be understood as the zeroth-order cumulant expansion in the quantum fluctuations. In doing so, the equation for the first moments (3a) forms already a closed set. The resulting equations are the celebrated DD-DNLS equations, in this case, with driving and dissipation:

$$
i d_{t} \varphi_{l}=\delta \omega \varphi_{l}+U\left|\varphi_{l}\right|^{2} \varphi_{l}-J\left(\varphi_{l+1}+\varphi_{l-1}\right)+A-\frac{i \gamma}{2} \varphi_{l} .
$$

For this set of equations [20,22], apart from dark solitons (localized minima in an intensity distribution) and sudden jumps from one intensity to another (kinks), there also exist bright solitons (localized intensity maxima) for a defocusing nonlinearity $U=-1$, on which we will concentrate in this work. The main question that we tackle is if the solutions found in the DD-DNLS survive the inclusion of quantum fluctuations.

\section{B. Second-order expansion (SOE)}

Let us consider now Eq. (3) up to second order of correlations. In doing so, we rewrite

$$
\hat{a}_{l}=\hat{a}_{l}-\left\langle a_{l}\right\rangle+\left\langle a_{l}\right\rangle=: \delta \hat{a}_{l}+\left\langle a_{l}\right\rangle .
$$


Neglecting terms with $\mathcal{O}\left(\delta \hat{a}^{3}\right) \simeq 0$, any $l$ correlator can be written in terms of two-point correlators:

$$
\begin{aligned}
\left\langle A_{1} A_{2} A_{3} A_{4}\right\rangle= & \sum_{\substack{j<k \\
l<m}}\left\langle A_{j} A_{k}\right\rangle\left\langle A_{l}\right\rangle\left\langle A_{m}\right\rangle \\
& -5\left\langle A_{1}\right\rangle\left\langle A_{2}\right\rangle\left\langle A_{3}\right\rangle\left\langle A_{4}\right\rangle,
\end{aligned}
$$

where $A_{j}$ can be any annihilation $a_{l}$ (creation $a_{l}^{\dagger}$ ) operator and $j \neq k \neq l \neq m$. Consequently, Eqs. (3a), (3b), and (3c) form a closed set which stands for a second-order expansion (SOE). It is worth emphasizing that, instead of the SOE, Gaussian expansions as the Hartree-Fock-Bogoliubov or higher order terms might also be considered [14,31,32]. For $U=0$, SOE and the Gaussian state approach have identical and exact solutions. However, in the parameter regime explored, our SOE is more stable than the Gaussian expansion [32] and approaches better the exact result for the single-site case [26]. Its numerical solution will be studied in Sec. IV B.

\section{Gutzwiller ansatz}

We will compare the results of SOE with the time evolution of a density matrix using a Gutzwiller ansatz [11,32]. This assumes a factorized form for the density matrix:

$$
\varrho=\prod_{l=1}^{L} \bigotimes \varrho_{l}
$$

with site-dependent density matrices $\varrho_{l}$. Using $\operatorname{tr}\left(\varrho_{l}\right)=1$ $\left(\operatorname{tr}\left(\partial_{t} \varrho_{l}\right)=0\right)$, we obtain the quantum nonlinear master equation set:

$$
\begin{aligned}
d_{t} \varrho_{l}= & -i\left[\delta \omega a_{l}^{\dagger} a_{l}+A\left(a_{l}^{\dagger}+a_{l}\right)+\frac{U}{2} a_{l}^{\dagger 2} a_{l}^{2}\right. \\
& \left.-J\left(\left(\left\langle a_{l+1}^{\dagger}\right\rangle+\left\langle a_{l-1}^{\dagger}\right\rangle\right) a_{l}+\text { H.c. }\right), \varrho_{l}\right] \\
& +\gamma a_{l} \rho_{l} a_{l}^{\dagger}-\frac{1}{2}\left\{a_{l}^{\dagger} a_{l}, \varrho_{l}\right\} .
\end{aligned}
$$

This ansatz considers fully the nonlinearity while it treats the hopping term as mean field.

\section{CIRCUIT-QED IMPLEMENTATION}

Although several systems may be modeled by means of a Bose-Hubbard model with losses and external driving as in Eqs. (1) and (2), we fix our attention on circuit-QED architectures [24].

The latter marks a technology based on superconducting circuits operating in $\mathrm{GHz}$ and cooled to $\mathrm{mK}$. Thus, the chip works in the quantum regime. Linear (transmission lines and resonators) and nonlinear (qubits) circuits can be coupled in a systematic and scalable way. Therefore, circuit-QED seems to be an ideal platform to study many-body physics [5]. In this subsection we argue that the fundamental blocks for simulating (1) already have been experimentally demonstrated.

The first ingredient is having nonlinear resonators. For that, we think about recent experiments where coplanar waveguide resonators are interrupted by a Josephson junction [cf. Fig. 1(c)]. The Josephson junction provides the nonlinearity through the term $E_{J} \cos \left(2 \pi / \Phi_{0} \delta \phi\right)$ in the effective action. Here $E_{J}$ is the Josephson energy, $\Phi_{0}$ the flux quanta, and $\delta \phi$ the jump in the flux at both sides of the junction [33,34]. In Ref. [35], the authors measured nonlinear resonators that can be modeled within the Hamiltonian (after expansion of the cosine):

$$
H=\omega_{0} a^{\dagger} a+\frac{U}{2}\left(a^{\dagger}\right)^{2} a^{2}+\frac{U^{\prime}}{2}\left(a^{\dagger}\right)^{3} a^{3}+\ldots,
$$

with $\omega_{0} \cong 6 \mathrm{GHz}$ and $U \cong-700 \mathrm{KHz}$. Please note that $U<0$. Therefore, by choosing pumps with driving frequencies detuned from $\omega_{0}$ in the $\mathrm{KHz}-\mathrm{MHz}$ regime different $U / \delta \omega$ in (1) can be simulated. Finally, higher order terms can be safely discarded, $U^{\prime} / U \cong 10^{-3}$. An intercavity coupling as in (1),

$$
H=J\left(a_{l}^{\dagger} a_{l-1}+\text { H.c. }\right) \text {, }
$$

already has been measured in a wide range of values for $J$, even reaching values of $J / \omega_{0} \cong 0.2$ [36]. Moreover, a tunable coupling $J$ has been achieved [37]. High reproducibility in the resonator bare frequencies, a necessary ingredient for building many-body arrays, also has been achieved [38].

Finally, a measurement scheme is mandatory. Here, we rely on the field tomography techniques developed in the circuit-QED community [39-41]. As explained in Sec. V, measuring field-field correlators is sufficient for accessing the different phases of (1), including the solitonic solutions. A possible architecture is depicted in Fig. 1(c). Inspired in Ref. [29] we envision a one-dimensional array of nonlinear cavities: superconducting resonators interrupted by a Josephson junction. The design is such that the coupling can be tuned by locally approaching the resonators [36]. The measurement can be accomplished by an auxiliar transmission line that couples the array and where an input field is impinged and the output is measured as we will explain in Sec. V. Therefore, the simulation and measurement may be possible within the technological state of the art.

\section{RESULTS}

We summarize here our numerical findings. We first review the classical DD-DNLS limit. Then, we report on our quantum results, both for the SOE and Gutzwiller ansatz.

\section{A. DD-DNLS: solitons with anticontinuous limit}

Imagine that two stable solutions exist for the single-site DD-DNLS, say $\varphi$ and $\varphi^{\prime}$. Then, at zero hopping $(J=0)$, the solution $\varphi_{m}=\varphi^{\prime}, \varphi_{l}=\varphi \forall l \neq m$ is a stable localized solution. This example corresponds to a soliton with a localized amplitude at one site $(m)$. This (trivial) soliton can be used as a starting point to find solutions by turning on the hopping, $J \neq 0$. The latter is a common procedure for finding localized modes in the DD-DNLS. In the nonlinear jargon, the zero hopping case is named as an anticontinuous limit and [6,7,42] a variety of numerical continuation techniques from zero to nonzero hopping have been used as, for example, the Newton-Raphson method. This procedure is not restricted to conservative settings, but also works very well in the driven dissipative classical models mentioned before. It was 


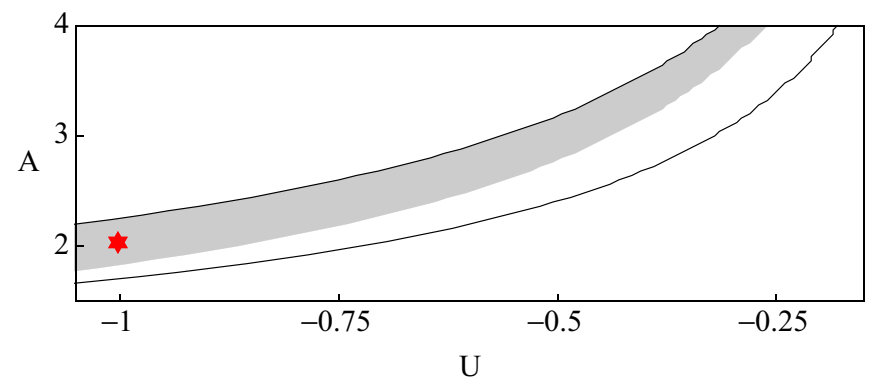

FIG. 2. (Color online) Number of single-site solutions vs $U$ and $A$ for the DD-DNLS and SOE approximations for $J=0$. Between the black lines, the DD-DNLS (4) has more than one solution (anticontinuous limit), whereas the solution for SOE is unique in the gray area. The red star denotes the parameters used along the manuscript.

used in [20,22] to characterize the localized modes for the DD-DNLS. In Fig. 2 the area between the two black lines shows, where the single-site solution of the DD-DNLS (4) is multivalued and therefore there exists an anticontinuous limit for localized modes. These results and the numerical continuation for nonzero coupling will be compared to SOE in the following subsection.

\section{B. SOE: Localization without anticontinuous limit}

In the quantum regime, the continuation from the zero hopping (anticontinuous limit) cannot be used. The reason is, that the single-site version of (1) and (2) has a unique solution [26]. Therefore, if quantum solitons exist they do not have an anticontinuous limit. Without the possibility of finding solitons by continuation, an educated guess is to try the search within the parameter regime where they exist in the classical DD-DNLS limit [20,22], thus in the area between the two black lines of Fig. 2. As anticipated, through this work we use the approximate treatment SOE. Therefore, for consistency, we must check that SOE has a unique solution in the single-site case $(J=0)$. In Fig. 2 we delimit this consistency region, which is shown in gray. The red star marks a point with a unique solution for the SOE but with proven solutions in the DD-DNLS, with $U=-1$ and $A=\gamma=2$. For a better comparison with the DD-DNLS [20,22], a detuning of $\delta \omega=3+2 J$ has been chosen. Therefore, $J$ remains the only free parameter. The latter set will be used along the text. However, we checked for different parameter sets within the gray area $[(U=-0.75, A=2.4)$ and $(U=-0.5, A=3)]$ and observed a similar scenario.

In general, steady-state solutions can be obtained by simply integrating the dynamics for (3) up to sufficiently long times such that a stationary dynamics is reached [43]. By construction, only stable solutions are found. Besides, there is no necessity for fine-tuned state preparation. Finally, this method provides not only steady-state solutions, but also time-periodic modes. Another possibility, which also finds unstable modes, is using the corresponding algebraic set of equations for $d_{t}\langle\ldots\rangle=0$ with, e.g., a Newton-Raphson scheme. Unfortunately, this method has no guarantee to converge and could be used successfully only for specific parameters (see the unstable soliton mentioned below). We use the long-time dynamics for all stable modes presented here.

Examples of different solutions assuming periodic boundary conditions are plotted in Fig. 3. In Fig. 3(a) the dynamics for a stationary ripple mode is depicted. In Figs. 3(b) and 3(c) examples for the time evolution of stationary and periodic localized modes are plotted, respectively. To visualize the physical mechanism yielding these solutions we choose to plot the mean between the minimum and maximum of the amplitudes $0.5\left(\min \left\{\left|\left\langle a_{l}(t)\right\rangle\right|\right\}+\max \left\{\left|\left\langle a_{l}(t)\right\rangle\right|\right\}\right)$ vs sites $l$ and $J$ in Fig. 3(d). This averaging is recommendable to better illustrate the localized character of the oscillatory mode; in all other regions of steady-state modes it does not change the picture. The figure shows that, for vanishing and small $J$, the homogeneous mode is the only stable solution. It becomes unstable at the value $J_{1} \simeq 0.1$, a symmetry-breaking bifurcation not present in the DD-DNLS limit. For small, but finite $J>0.1$ the ripple modes with one site having a higher amplitude than its two neighbors dominate the dynamics. For the spatial periodicity of these modes there is a certain dependency on the number of sites $L$, as can be seen in Fig. 3(d) with $L=25$ leading to a defect in the right bottom corner, whereas for $L=30 \mathrm{in} \mathrm{Fig.} \mathrm{3(b)} \mathrm{no} \mathrm{such} \mathrm{defect} \mathrm{can} \mathrm{be} \mathrm{found.} \mathrm{As}$ the coupling increases, repeated bifurcations into modes with different periodicity can be observed as the extension of the maxima grows and fewer peaks can be accommodated within the lattice. Finally, this leads to only one central localized mode in Fig. 3(d) at $J_{2}$ appearing dynamically as the steady state. Increasing $J$, the stationary soliton starts to oscillate at $J_{3}$ and finally relaxes to the homogeneous mode for $J_{4} \gtrsim 5.38$. Comparing the qualitative behavior of the SOE to its classical limit in the DD-DNLS, we observe that for vanishing hopping the SOE only has one homogeneous solution, which is in accordance with the exact quantum limit. Then, the ripple mode appears introducing a periodic variation of the amplitude distribution. Similar variations can also be found in the exact solution of driven Bose-Hubbard chains with dissipation at the ends [44]. There, it is interpreted as a protection of a sublattice from the coherent pumping. As the hopping increases, the amplitudes and width of the maxima grow and therefore less maxima can be accommodated, and the ripple periodicity changes abruptly leading finally to the localization into only one maximum. The SOE thus does exhibit various periodicity changing bifurcations towards more and more localization, a behavior not found in the DD-DNLS. Increasing $J$ further, the localized mode becomes unstable and starts to oscillate. For large $J$ the solution should be represented by a mean-field limit, which considers only an effective perturbation due to the coupling to neighboring sites, thus a homogeneous solution. Our findings are thus in very good accordance with the mean-field theory.

We have seen that the classical DD-DNLS is a particular limit of the quantum model in which the quantum fluctuations are neglected. If the quantum corrections were negligible, both the SOE and the DD-DNLS would produce similar results. As shown in Fig. 3(e) this is not the case. There we plot the dependence of the center-site amplitude $\left|\left\langle a_{c}\left(t_{\text {evol }}\right)\right\rangle\right|$ on $J$ after the dynamics settled into a steady state or periodic state of SOE (3) (red) within the evolution time $t_{\text {evol }}=100$. Please note, that all possible phases are shown in Fig. 3(e) 

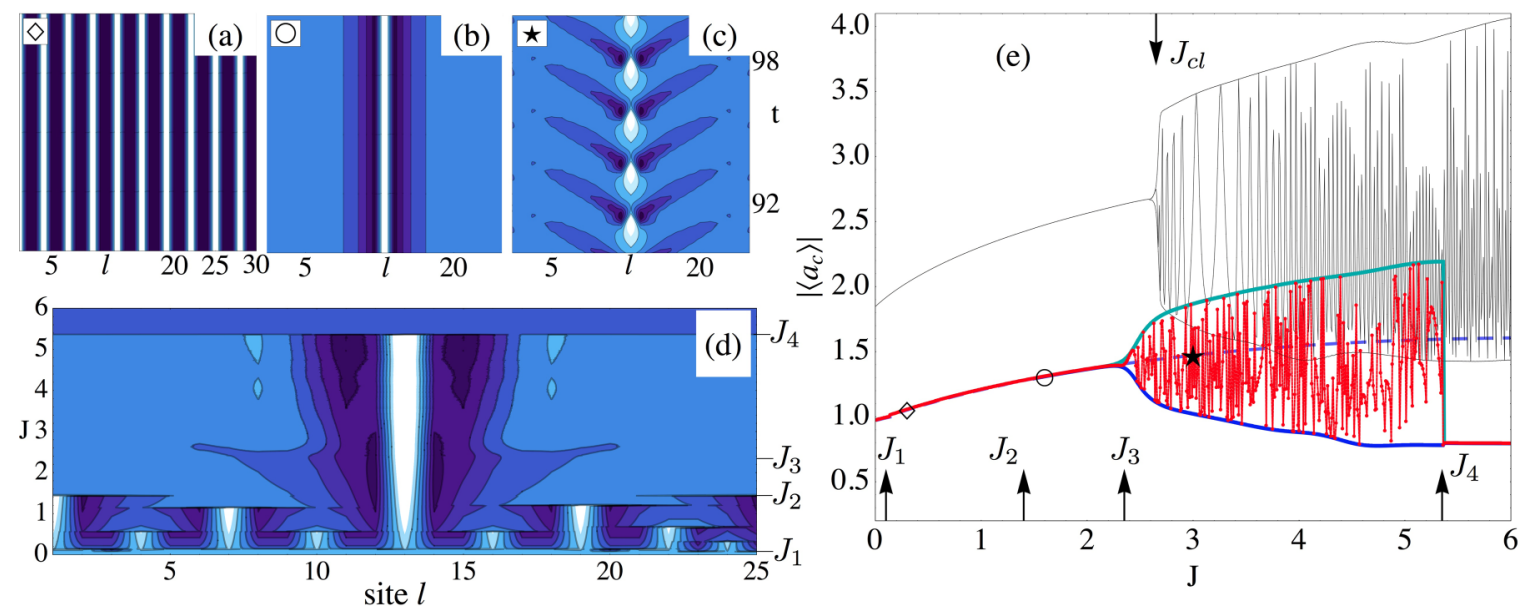

FIG. 3. (Color online) Long-time dynamics of $\left|\left\langle a_{l}\right\rangle\right|$ vs site $l$ and time $t$ under SOE (3), showing ripples for $J=0.3$ (a), a stable soliton for $J=1.6(\mathrm{~b})$, and the oscillatory localized mode for $J=3(\mathrm{c})$. (d) Mean between the minimum and maximum of the amplitudes $\left[0.5\left(\min \left\{\left|\left\langle a_{l}(t)\right\rangle\right|\right\}+\max \left\{\left|\left\langle a_{l}(t)\right\rangle\right|\right\}\right)\right]$ vs sites $l$ and $J$ for the SOE steady state. (e) Amplitude of the center site for DD-DNLS (thin black), SOE Max(thick green upper branch), SOE Min (thick blue lower branch), and SOE fixed integration time (thick red) and unstable localized stationary mode (dashed blue line).

and that the value of $\left|\left\langle a_{c}\right\rangle\right|$ does not necessarily indicate that there is a difference to neighboring sites. The arrows point to the bifurcation points $J_{i}$. When the dynamics is determined by the periodic mode we also show the maximum and minimum of $\left|\left\langle a_{c}(t)\right\rangle\right|$ in green and blue. The algebraic stationary and unstable localized mode is shown with a blue dashed line. For comparison we show the results of the classical limit (4) in black, also exhibiting a periodic mode, but for higher $J$. The classical amplitude is nearly twice as high as the SOE value, but the main difference is in the classes of solutions found. Whereas the soliton mode is stable in the classical DD-DNLS limit from $J=0$ up to the appearance of the periodic solution at $J_{\mathrm{cl}}$, for the SOE limit there is no anticontinuous limit. At $J_{1}$ the ripples appear and persist for $J_{1}<J<J_{2}$. The bifurcation into the periodic solution is located at $J_{3}<J_{\mathrm{cl}}$, as well as the high-coupling homogenous mode at $J_{4}$. The symbols in the SOE families denote the examples shown in Figs. 3(a)-3(c).

\section{Gutzwiller ansatz}

We complement the SOE with the dynamics within the Gutzwiller ansatz. (7). As initial conditions, we use the homogeneous steady state for the corresponding value of $J$ at all sites but the center, where we assume a coherent state with a higher number operator $\left\langle a_{c}^{\dagger}(t=0) a_{c}(t=0)\right\rangle \equiv\left\langle n_{c}(t=\right.$ $0)\rangle \simeq 8.8$. The Fock space per lattice site is truncated to a maximum of 15 excitations in a lattice of 15 sites. Within this parameter space, we were able to find a region at $J \simeq 2$, where the initially localized distribution survives much longer. Even though we can compare the SOE and the Gutzwiller ansatz only qualitatively, this corresponds to the regime of stable soliton modes in the SOE limit. In Fig. 4(a) we show the time evolution of $\Delta n_{c, 1}(t)=\left\langle n_{c}(t)\right\rangle-\left\langle n_{1}(t)\right\rangle$ vs $t$ and $J$, which gives indications about the survival time of localization. Whereas for small $J$ the value of $\Delta n_{c, 1}(t)$ decays very fast, the relaxation to the homogeneous state for $J \simeq 2$ is much slower. The values of $\left\langle n_{l}\right\rangle$ in the whole array for $J=3.5$ and $J=2$ are plotted in Figs. 4(b) and 4(c), respectively. Figure 4(c) shows that the initial value decays abruptly to $n_{c} \simeq 3$, and from that point on, the decay becomes very slow. This indicates the existence of a weakly unstable localized mode. To show the parallels with the behavior of the static localized modes of the SOE existing between the values $J_{2}$ and $J_{3}$ we also indicate them here. For the example shown in Fig. 4(b), the initial decay is equally fast, furthermore, some oscillations can be observed, which is a reminiscence of the existence of the periodic mode in the SOE limit for these parameter values. Additionally, hints of these oscillations can be seen in $\Delta n_{c, 1}$ for $J \simeq 3.5$. We could not find any indications of ripple modes, since they present higher-order intersite correlations neglected within the Gutzwiller ansatz, as we will show in the following section.

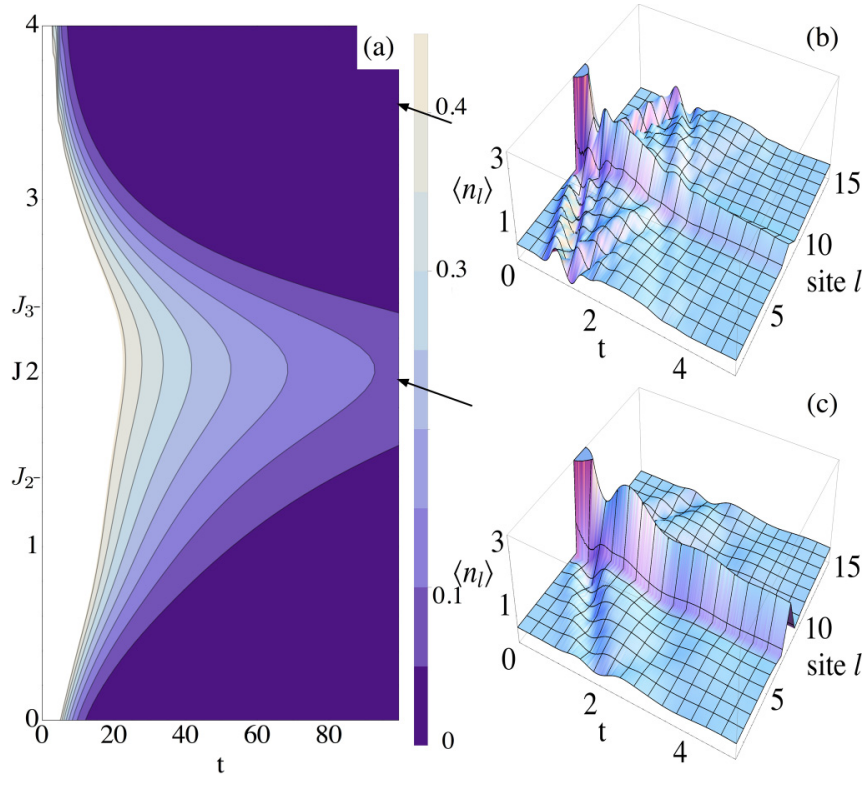

FIG. 4. (Color online) Gutzwiller ansatz (8): (a) $\left\langle n_{c}(t)\right\rangle-$ $\left\langle n_{1}(t)\right\rangle$. (Left) $\left\langle n_{l}(t)\right\rangle$ for $J=2$ (b) and $J=3.5$ (c). 


\section{IN-OUT MECHANISM}

The question still remains of how to extract the information stored in our discrete array of cavities. In order to do this, we will follow the input-output formalism [45]. The basic idea here is to make our dissipative system interact with the electromagnetic field, in the form of a transmission line [cf. Figs. 1(b) and 1(c)]. The electromagnetic field can be decomposed into two contributions: the input, or the radiation impinging onto the system, and the output, the sum of a reflected plus a radiated component [see Fig. 1(b)]. The latter is determined by the system and its interaction with the transmission line. Therefore, measuring the output (and comparing it with the input) we can infer information on the system dynamics.

We will briefly sketch the main steps to derive the inputoutput relations. Details on the calculations can be found in Appendix A (see also the Supplemental Material of Ref. [46]). The system we want to acquire information from is an open system described by the Hamiltonian (1) and the master equation (2). Let us denote this open system: the Bose-Hubbard model + dissipation and driving as $H_{\text {open }}$.

The open system $H_{\text {open }}$ is coupled to a transmission line as depicted in Fig. 1(c). The total Hamiltonian, including the transmission line and the interaction of our system with it, reads $H=H_{\text {open }}+H_{\mathrm{TL}}+H_{\text {int }}$. The transmission line interacts directly with the cavities and it can be viewed as a onedimensional electromagnetic field. In second quantization, the electromagnetic field can be described as a collection of harmonic oscillators. As it is depicted in Fig. 1(b), we should consider carefully the direction of propagation of excitations in the transmission line. For this task we will introduce the electromagnetic field operators: $l(p)\left(l^{\dagger}(p)\right)$ which destroys (creates) a photon with momentum $p$ propagating to the left and $r(p)\left(r^{\dagger}(p)\right)$ which destroys (creates) a photon with momentum $p$ propagating to the right. In terms of these and for a linear dispersion relation $\left(\omega_{p}=v|p|\right)$, the Hamiltonian of the electromagnetic field in the transmission line reads

$$
H_{\mathrm{TL}}=v\left(\int_{0}^{\infty} d p v p r^{\dagger}(p) r(p)-\int_{-\infty}^{0} d p v p l^{\dagger}(p) l(p)\right),
$$

with $v$ the speed of light in the transmission line. Finally, the interaction $H_{\text {int }}$ considers the most general type of coupling in a solid-state device. It consists of an inductive part (flux interaction) and a capacitive contribution (charge interaction). The interactions are weak and pointlike, happening at the position of every cavity, yielding

$$
\begin{aligned}
H_{\text {int }} \sim & \frac{i g}{\sqrt{2 \pi L}} \sum_{k}\left(\int_{+k} \frac{d p}{\sqrt{\omega_{p}}} L\left(r^{\dagger}(p) a_{k}(t)-\text { H.c. }\right)\right. \\
& \left.+\int_{-k} \frac{\mathrm{d} p}{\sqrt{\omega_{p}}} L\left(l^{\dagger}(p) a_{k}(t)-\text { H.c. }\right)\right)
\end{aligned}
$$

where we have introduced a plane wave expansion for the cavity operators,

$$
a_{l}(t)=\frac{1}{\sqrt{L}} \sum_{k} e^{i k x_{l} / d} a_{k}(t),
$$

$d$ being the lattice spacing of our cavity array with $N$ sites. We are now able to solve the Heisenberg equations for the left and right operators. The idea is to relate the contributions of all momenta before the interaction (from an initial time $t_{0} \rightarrow-\infty$ ) and after the interaction (up to a time $t_{1} \rightarrow \infty$ ). As it is shown in Appendix A, for every cavity momentum $k$ we only have significant contributions from those momenta $p$ in a narrow region around the former. For the right operators, we call this momentum interval $+k$ and for the left ones $-k$ [cf. Eq. (A10)]. Taking this into account, we introduce the (right) input operator,

$$
r_{\text {in }}^{+k}(t)=\frac{1}{\sqrt{2 \pi}} \int_{+k} d p e^{-i v p\left(t-t_{0}\right)} r_{0}(p),
$$

for times $t>t_{0}\left(r_{0}\right.$ denotes the $r$ operator at $\left.t=t_{0}\right)$ and the (right) output operator,

$$
r_{\text {out }}^{+k}(t)=\frac{1}{\sqrt{2 \pi}} \int_{+k} d p e^{-i v p\left(t-t_{1}\right)} r_{1}(p),
$$

for times $t<t_{1}\left(r_{1}\right.$ denotes the $r$ operator at $\left.t=t_{1}\right)$. And similarly for the $l$ operators. Thus, the Heisenberg equations lead to the following input-output relations,

$$
\begin{aligned}
& r_{\text {out }}^{+k}(t)=r_{\text {in }}^{+k}(t)+\frac{1}{d} \sqrt{\frac{\Gamma v}{L k}} e^{-i k v t / d} a_{k}, \\
& l_{\text {out }}^{-k}(t)=l_{\text {in }}^{-k}(t)+\frac{1}{d} \sqrt{\frac{\Gamma v}{L k}} e^{+i k v t / d} a_{k},
\end{aligned}
$$

with $\Gamma$ a constant characterizing the strength of the transmission line-nonlinear cavity coupling, and $a_{k}$ defined as

$$
a_{k}=\frac{1}{\sqrt{2 \pi}} \int d t^{\prime} e^{i k v t^{\prime} / d} a_{k}\left(t^{\prime}\right)
$$

In the presence of the transmission line the equations of motion for the $a_{k}(t)$ operators differ from those obtained from (2). The transmission line plays now the role of a second environment for the system described by (1). However, under the same approximations which led to (2) (Markov approximation), we immediately see that the role of the transmission line is to renormalize the decay rates $\gamma$. Namely, to add a contribution proportional to $g^{2}$ to them. In addition, the input field will renormalize the driving field amplitude (with strength of order $g$ ).

Within relations (16) and (17) we can map output fieldfield correlations to cavity mode correlations. For example, using relation (16) $\left\langle r_{\text {out }}^{+k \dagger}\left(t_{1}\right) r_{\text {out }}^{+k}\left(t_{2}\right)\right\rangle$ gives us information on the system two-point correlator $\left\langle a_{k}^{\dagger} a_{k^{\prime}}\right\rangle$ provided that we only impinge a signal from the left,

$$
\left\langle r_{\text {out }}^{+k \dagger}\left(t_{1}\right) r_{\text {out }}\left(t_{2}\right)\right\rangle=\frac{1}{d^{2}}\left(\frac{\Gamma v}{L \sqrt{k k^{\prime}}}\right) e^{i v\left(k t_{1}-k^{\prime} t_{2}\right) / d}\left\langle a_{k}^{\dagger} a_{k^{\prime}}\right\rangle .
$$

Similar relations hold for other two-point correlations.

Therefore, two time correlations in the output field map to the system two point correlations in momentum space. In other words, within our proposed setup, the experimentally accessible quantities are correlators in momentum, rather than in position. It remains, then, to check if these are also useful for distinguishing the different phases discussed here. For that, 


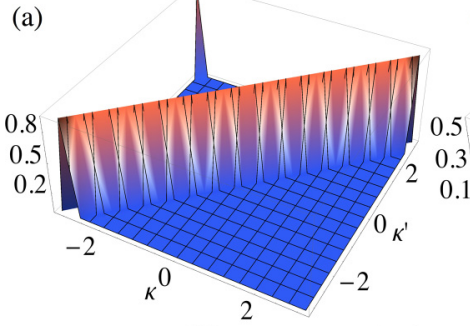

(b)

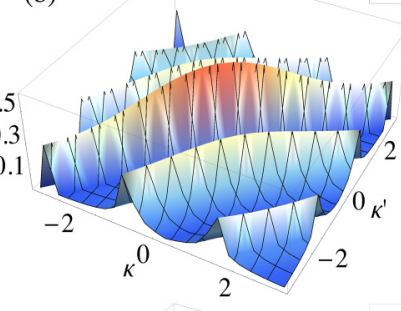

(c)

(d)
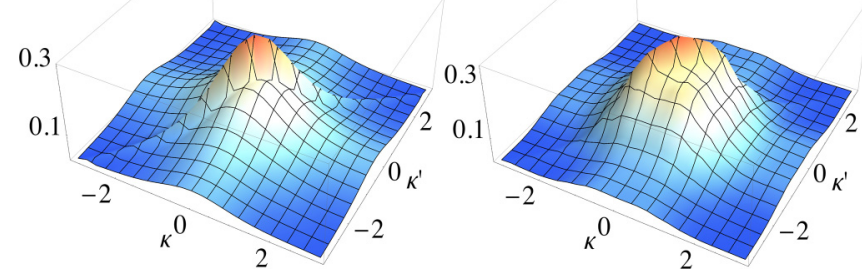

FIG. 5. (Color online) Connected correlator $f\left(a_{k}, a_{k^{\prime}}^{\dagger}\right)$ (20) for $J=0,(0.3,1.6,3)$ (a)-(d). For (b)-(d), the symbols correspond to the parameter values and solutions shown in Figs. 3(a)-3(c).

we introduce the connected correlator,

$$
f(a, b)=\langle a b\rangle-\langle a\rangle\langle b\rangle .
$$

It is worth emphasizing that the above is identically zero in the classical limit. Therefore, the connected correlator indicates the presence of fluctuations at the quantum level. In Fig. 5 we plot $f\left(a_{k}, a_{k^{\prime}}^{\dagger}\right)$ for different stationary SOE solutions. The homogeneous solution $(J=0)$ is shown in Fig. 5(a). The ripples $(J=0.3)$ are drawn in Fig. 5(b) with the same parameters as in Fig. 3(a). Localized solutions, static and oscillatory, are given in Figs. 5(c) and 5(d), respectively. Their real space counterparts were given in Figs. 3(b) and 5(d). The main difference between (20) for the static and oscillatory localized mode is that the connected correlator of Fig. 5(d) strongly oscillates in time. The differences in magnitude of the maxima are nearly of a factor two, whereas Fig. 5(c) is static in time. The momentum spaces for each phase are clearly distinguishable from each other and show, that the input-output mechanism presents a perfect measurement scheme to prove the existence and stability of localized modes.

\section{DISCUSSION}

The phases for the Bose-Hubbard model with gain and loss have been investigated within a semiclassical approach. It has been argued that, in the zero hopping case, the unique solution is the homogeneous one. A translational-invariant broken symmetry solution (ripples) appears when the hopping term reaches some critical value. Increasing the hopping, the extension of the ripples grows and their periodicity decreases in a second bifurcation. Eventually, the discrete periodicity disappears and only one maximum remains, the stable localized mode. Passing from static to periodic (in time), this mode finally becomes unstable at higher $J$, transiting to a homogenous solution.

The symmetry-breaking transition from homogenous to discrete periodic, the bifurcations to localized static and periodic modes, and the symmetry-breaking transition back to homogenous mark novel phases without a counterpart in the Hamiltonian limit (zero dissipation, zero gain) of the BoseHubbard where the well-known Mott-superfluid transition has been largely described.

Apart from the interest in finding novel matter phases in the many-body phase diagram, artificial systems with driving and dissipation present also a natural way of observing localization.

Our calculations rely on a semiclassical approximation. We have complemented them with a Gutzwiller ansatz where the onsite dynamics is expected to be more accurate but intersite correlations are poorly described. Nevertheless, the regions where solitons exist in the semiclassical regime present long-lived localized solutions within the Gutzwiller ansatz. Therefore, we expect that the semiclassical phases have some traces in the full, not yet explored, quantum dynamics.

The richness of phases presented here may be a motivation for future works considering the full quantum aspects of the model. In this line, our proposal within circuit-QED presents a quantum simulator for going beyond the theory presented here.

\section{ACKNOWLEDGMENTS}

We acknowledge support from the Spanish DGICYT under Projects No. FIS2012-33022 and No. FIS2011-25167 as well as the Aragón (Grupo FENOL) and the EU Project PROMISCE.

\section{APPENDIX A: INPUT-OUTPUT THEORY}

Here we derive the input-output relations for the dissipative driven Bose-Hubbard model coupled to a transmission line. The total Hamiltonian reads

$$
H=H_{\text {open }}+H_{\mathrm{TL}}+H_{\text {int }},
$$

where $H_{\text {open }}$ accounts for the open system: Bose-Hubbard model + driving + environment. For simplicity, we will refer to the latter as our system. $H_{\mathrm{TL}}$ describes the electromagnetic field propagating through the TL. In momentum space it is given by

$$
H_{\mathrm{TL}}=v\left(\int_{0}^{\infty} d p v p r^{\dagger}(p) r(p)-\int_{-\infty}^{0} d p v p l^{\dagger}(p) l(p)\right),
$$

where we are assuming a linear dispersion relation $\omega_{p}=v|p|$. Our cQED proposal involves impinging a signal into the system and gathering information about it by means of the reflected and transmitted components of the former. For this task, it is helpful to decompose the electromagnetic field operators in $l^{\dagger}(p)(l(p))$ which creates (annihilates) an excitation with momentum $p$ propagating to the left with velocity $v$ (the speed of light in the transmission line) and similarly $\left.r^{\dagger}(p)\right) r(p)$ ), which creates (annihilates) an excitation with momentum $p$ propagating to the right with velocity $v$. Finally, $H_{\text {int }}$ is the interaction Hamiltonian,

$$
H_{\text {int }}=i \sum_{n} \int d x g_{n}(x) \hat{A}(x)\left(a_{l}-a_{l}^{\dagger}\right) .
$$


Here we are considering a generic coupling between a system operator and the electromagnetic potential $\hat{A}(x)$ :

$$
\begin{aligned}
\hat{A}(x)= & v\left(\int_{-\infty}^{0} d p \sqrt{\frac{1}{2 \pi \omega_{p}}} l(p) e^{i p x}\right. \\
& \left.+\int_{0}^{+\infty} d p \sqrt{\frac{1}{2 \pi \omega_{p}}} r(p) e^{i p x}+\text { H.c. }\right) .
\end{aligned}
$$

In [46] following the lumped circuit element description of a transmission line, we decomposed the interaction into capacitive and inductive contributions. The latter are encoded in the coupling function $g_{n}(x)$. We now introduce a plane wave expansion for the cavity operators $a_{l}$,

$$
a_{l+1}(t)=\frac{1}{\sqrt{N}} \sum_{k} e^{i k x_{n} / d} a_{k}(t),
$$

where $d$ is the lattice spacing of our cavity array with $N$ sites. Assuming a rotating wave approximation (RWA) regime we can rewrite (A3) as

$$
\begin{aligned}
H_{\text {int }}= & \frac{i v}{\sqrt{2 \pi N}} \sum_{k, n} \\
& \times\left[\int_{-\infty}^{0} \frac{d p}{\sqrt{\omega_{p}}} \int_{-\infty}^{+\infty} d x g_{n}(x) e^{-i\left(p x-\frac{k x_{n}}{d}\right)} l^{\dagger}(p) a_{k}\right. \\
& +\int_{0}^{+\infty} \frac{d p}{\sqrt{\omega_{p}}} \int_{-\infty}^{+\infty} d x g_{n}(x) e^{-i\left(p x-\frac{k x_{n}}{d}\right)} r^{\dagger}(p) a_{k} \\
& - \text { H.c. }] .
\end{aligned}
$$

The transmission line only couples to the system at the position of the cavities, therefore, $g_{n}(x)=g \delta\left(x-x_{n}\right)$ and we have

$$
\begin{aligned}
H_{\text {int }}= & \frac{i v g}{\sqrt{2 \pi N}} \sum_{k}\left(\int_{-\infty}^{0} \frac{d p}{\sqrt{\omega_{p}}} \sum_{n}\left(l^{\dagger}(p) a_{k} e^{-i\left(\frac{\omega_{p}}{v}+\frac{k}{d}\right) x_{n}}\right)\right. \\
& \left.+\int_{0}^{+\infty} \frac{d p}{\sqrt{\omega_{p}}} \sum_{n}\left(r^{\dagger}(p) a_{k} e^{-i\left(\frac{\omega_{p}}{v}-\frac{k}{d}\right) x_{n}}\right)-\text { H.c. }\right),
\end{aligned}
$$

where we have replaced $p$ in the exponentials for $\pm \omega_{p} / v$.

We can approximate the sum,

$$
\sum_{n} e^{-i\left(\omega_{p} / v-k / d\right) x_{n}}
$$

by a rectangle of height $N$ and width $2 \delta=2 \pi / N$ centered at $k$ (being zero elsewhere) (see Fig. 6). Similarly, $\sum_{n} e^{i\left(\omega_{p} / v+k / d\right) x_{n}}$ will be replaced by a rectangle centered in $-k$. Therefore, we can rewrite (A7) as

$$
\begin{gathered}
H_{\text {int }} \sim \frac{i v g}{\sqrt{2 \pi N}}\left(\int_{+k} \frac{d p}{\sqrt{\omega_{p}}} N\left(r^{\dagger}(p) a_{k}-\text { H.c. }\right)\right. \\
\left.+\int_{-k} \frac{d p}{\sqrt{\omega_{p}}} N\left(l^{\dagger}(p) a_{k}-\text { H.c. }\right)\right)
\end{gathered}
$$

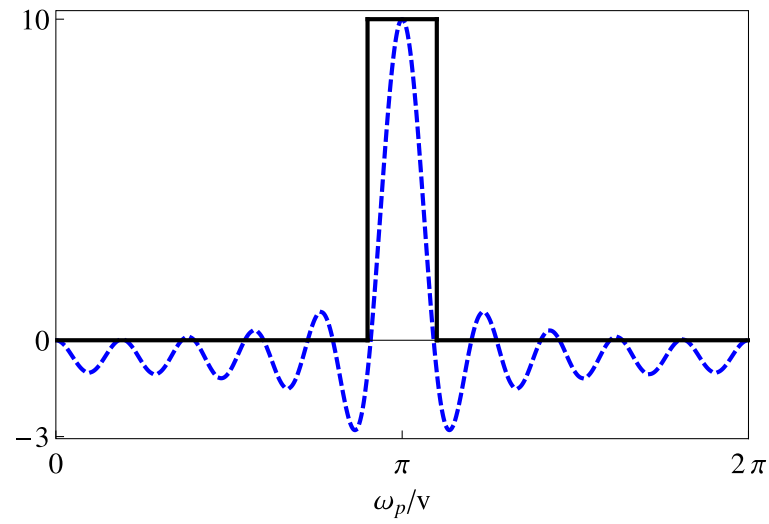

FIG. 6. (Color online) Sum (A8) (dashed) as a function of $\omega / d$ for $N=10$, and $k / d=\pi$. Rectangle approximation of (A8) (solid line). This considers that the function is different from zero only in the region between its first two zeros (symmetric around $k / d$ ) and that the height is constant and equal to $N$.

where the integration intervals, following our previous approximation, are

$$
\pm k \equiv[( \pm k-\delta) / d,( \pm k+\delta) / d] .
$$

We are now able to write the Heisenberg equation of motion for the electromagnetic field operators. Following (A1), (A2), and (A9) they are

$$
\begin{aligned}
& \dot{r}(p)=-i v p r(p)+v g \sqrt{\frac{N}{2 \pi \omega_{p}}} a_{k}(t), \\
& \dot{i}(p)=+i v p l(p)+v g \sqrt{\frac{N}{2 \pi \omega_{p}}} a_{k}(t) .
\end{aligned}
$$

Here we have included the explicit time dependence of the momentum cavity operators $a_{k}$. Integrating (A11) from $t_{0}$ to $t$ $\left(t_{0}<t\right)$ yields

$$
\begin{aligned}
r(p, t)= & e^{-i v p\left(t-t_{0}\right)} r_{0}(p) \\
& +v g \sqrt{\frac{N}{2 \pi \omega_{p}}} \int_{t_{0}}^{t} d t^{\prime} e^{-i v p\left(t-t^{\prime}\right)} a_{k}\left(t^{\prime}\right),
\end{aligned}
$$

where $r_{0}$ denotes the $r$ operator at time $t=t_{0}$. Notice that the former equations include a continuous momentum $p$ and a discrete one $k$. Recall from our approximation to the sum (A8) that for any given $k$ only the momenta $p$ in a very narrow region of width $\delta$ (centered in $k$ ) contribute to our expressions. We now integrate (A13) over this momentum interval $(+k$ for the right operators),

$$
\begin{aligned}
\int d p r(p, t) & \\
= & \sqrt{2 \pi} r_{i n}^{+k}(t)+g \sqrt{\frac{N v}{2 \pi k}} \int_{t_{0}}^{t} d t^{\prime} \\
& \times\left(\frac{2}{v\left(t-t^{\prime}\right)} e^{-i k v\left(t-t^{\prime}\right) / d} \sin \left(\frac{\delta v\left(t-t^{\prime}\right)}{d}\right) a_{k}\left(t^{\prime}\right)\right),
\end{aligned}
$$




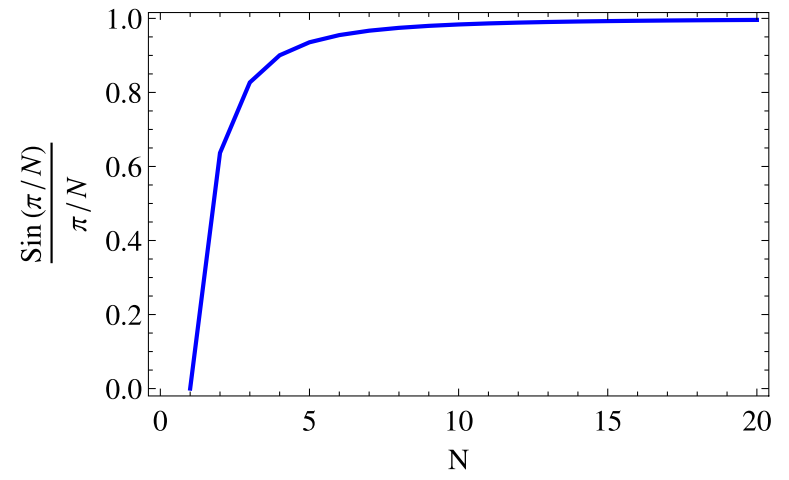

FIG. 7. (Color online) $\frac{\sin (y \pi / N)}{y \pi / N}$ as a function of the number $N$

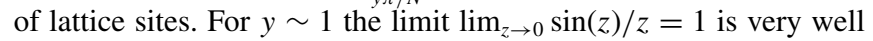
approximated with a few number of sites $(N \sim 10)$.

with the input operator $r_{\text {in }}^{+k}$ defined as (following [45])

$$
r_{\text {in }}^{+k}(t)=\frac{1}{\sqrt{2 \pi}} \int_{(k-\delta) / d}^{(k+\delta) / d} d p e^{-i v p\left(t-t_{0}\right)} r_{0}(p) .
$$

We have included the super index $+k$ to stress that this operator sums the momentum contributions in a narrow band around $k$ (positive for the right operators). The input operator takes into account the free evolution of all right-propagating electromagnetic field modes before the interaction with the system. Therefore, it acts as a driving field in the equations of motion of the cavity operators. In the continuum limit $(\delta \rightarrow 0)$ and for $t_{0} \rightarrow-\infty,(\mathrm{A} 14)$ yields

$$
\int d p r(p, t)=\sqrt{2 \pi}\left(r_{i n}^{+k}(t)+\frac{g}{d} \sqrt{\frac{\pi v}{2 N k}} e^{-i k v t / d} a_{k}\right) .
$$

As $\delta \rightarrow 0$, we make use of the following limit: $\lim _{z \rightarrow 0} \sin (z) / z=1$ for $z=\delta v\left(t-t^{\prime}\right) / d$. This holds reasonably well for $N \gtrsim 10$ as it is shown in Fig. 7. In addition, we have introduced

$$
a_{k}=\frac{1}{\sqrt{2 \pi}} \int_{-\infty}^{+\infty} d t^{\prime} e^{i k v t^{\prime} / d} a_{k}\left(t^{\prime}\right)
$$

In a similar way, we can integrate (A11) from $t$ to $t_{1}\left(t<t_{1}\right)$ and define a corresponding output operator.

$$
r_{\text {out }}^{+k}(t)=\frac{1}{\sqrt{2 \pi}} \int_{+k} d p e^{-i v p\left(t-t_{1}\right)} r_{1}(p) .
$$

We will find that the input and output operators are related by

$$
r_{\text {out }}^{+k}(t)=r_{\text {in }}^{+k}(t)+\frac{1}{d} \sqrt{\frac{\Gamma v}{N k}} e^{-i k v t / d} a_{k},
$$

while for the left operators we find

$$
l_{\text {out }}^{-k}(t)=l_{\text {in }}^{-k}(t)+\frac{1}{d} \sqrt{\frac{\Gamma v}{N k}} e^{+i k v t / d} a_{k},
$$

where we have chosen conveniently $g=\sqrt{\Gamma / 2 \pi}$.
[1] O. Morsch and M. Oberthaler, Rev. Mod. Phys. 78, 179 (2006).

[2] R. Blatt and C. F. Roos, Nat. Phys. 8, 277 (2012).

[3] U. Naether, J. J. García-Ripoll, J. J. Mazo, and D. Zueco, Phys. Rev. Lett. 112, 074101 (2014).

[4] J. Raftery, D. Sadri, S. Schmidt, H. E. Türeci, and A. A. Houck, Phys. Rev. X 4, 031043 (2014).

[5] A. A. Houck, H. E. Türeci, and J. Koch, Nat. Phys. 8, 292 (2012).

[6] P. Kevrekidis, Springer Tracts in Modern Physics (Springer, Berlin/Heidelberg, 2009).

[7] S. Flach and A. Gorbach, Phys. Rep. 467, 1 (2008).

[8] F. Lederer and G. Stegeman, Phys. Rep. 463, 1 (2008).

[9] Y. Kartashov, B. Malomed, and L. Torner, Rev. Mod. Phys. 83, 247 (2011).

[10] U. Naether, A. J. Martínez, D. Guzmán-Silva, M. I. Molina, and R. A. Vicencio, Phys. Rev. E 87, 062914 (2013).

[11] K. V. Krutitsky, J. Larson, and M. Lewenstein, Phys. Rev. A 82, 033618 (2010).

[12] R. V. Mishmash, I. Danshita, C. W. Clark, and L. D. Carr, Phys. Rev. A 80, 053612 (2009).

[13] A. D. Martin and J. Ruostekoski, Phys. Rev. Lett. 104, 194102 (2010).

[14] D. Witthaut, F. Trimborn, H. Hennig, G. Kordas, T. Geisel, and S. Wimberger, Phys. Rev. A 83, 063608 (2011).

[15] S. Burger, K. Bongs, S. Dettmer, W. Ertmer, K. Sengstock, A. Sanpera, G. V. Shlyapnikov, and M. Lewenstein, Phys. Rev. Lett. 83, 5198 (1999).

[16] K. E. Strecker, G. B. Partridge, A. G. Truscott, and R. G. Hulet, Nature (London) 417, 150 (2002).
[17] D. J. Frantzeskakis, J. Phys. A 43, 213001 (2010).

[18] H. Landa, S. Marcovitch, A. Retzker, M. B. Plenio, and B. Reznik, Phys. Rev. Lett. 104, 043004 (2010).

[19] M. Mielenz, J. Brox, S. Kahra, G. Leschhorn, M. Albert, T. Schaetz, H. Landa, and B. Reznik, Phys. Rev. Lett. 110, 133004 (2013).

[20] U. Peschel, O. Egorov, and F. Lederer, Opt. Lett. 29, 1909 (2004)

[21] J. E. Prilepsky, A. V. Yulin, M. Johansson, and S. A. Derevyanko, Opt. Lett. 37, 4600 (2012).

[22] O. A. Egorov and F. Lederer, Opt. Lett. 38, 1010 (2013)

[23] M. Johansson, J. E. Prilepsky, and S. A. Derevyanko, Phys. Rev. E 89, 042912 (2014).

[24] J. Jin, D. Rossini, R. Fazio, M. Leib, and M. J. Hartmann, Phys. Rev. Lett. 110, 163605 (2013).

[25] J. Jin, D. Rossini, M. Leib, M. J. Hartmann, and R. Fazio, Phys. Rev. A 90, 023827 (2014).

[26] P. Drummond and D. Walls, J. Phys. A 13, 725 (1980).

[27] A. Le Boité, G. Orso, and C. Ciuti, Phys. Rev. Lett. 110, 233601 (2013).

[28] A. L. Boité, G. Orso, and C. Ciuti, Phys. Rev. A 90, 063821 (2014)

[29] M. Leib, F. Deppe, A. Marx, R. Gross, and M. J. Hartmann, New J. Phys. 14, 075024 (2012).

[30] A. Rivas and S. F. Huelga, Open Quantum Systems. An Introduction, Springer Briefs in Physics (Springer, Berlin/Heidelberg, 2011). 
[31] I. Tikhonenkov, J. R. Anglin, and A. Vardi, Phys. Rev. A 75, 013613 (2007).

[32] F. Quijandría, U. Naether, D. Porras, J. J. García-Ripoll, and D. Zueco, J. Phys. B 48, 55302 (2015).

[33] D. Zueco, J. J. Mazo, E. Solano, and J. J. García-Ripoll, Phys. Rev. B 86, 024503 (2012).

[34] J. Bourassa, F. Beaudoin, J. M. Gambetta, and A. Blais, Phys. Rev. A 86, 013814 (2012).

[35] F. R. Ong, M. Boissonneault, F. Mallet, A. Palacios-Laloy, A. Dewes, A. C. Doherty, A. Blais, P. Bertet, D. Vion, and D. Esteve, Phys. Rev. Lett. 106, 167002 (2011).

[36] M. Haeberlein, D. Zueco, P. Assum, T. Weißl, E. Hoffmann, B. Peropadre, J. García-Ripoll, E. Solano, F. Deppe, A. Marx, and R. Gross, arXiv:1302.0729.

[37] A. Baust, E. Hoffmann, M. Haeberlein, M. J. Schwarz, P. Eder, J. Goetz, F.Wulschner, E. Xie, L. Zhong, F. Quijandría, B. Peropadre, D. Zueco, J.-J. García-Ripoll, E. Solano, K. Fedorov, E. P. Menzel, F. Deppe, A. Marx, and R. Gross, Phys. Rev. B 91, 014515 (2015).
[38] D. L. Underwood, W. E. Shanks, J. Koch, and A. A. Houck, Phys. Rev. A 86, 023837 (2012).

[39] E. P. Menzel, F. Deppe, M. Mariantoni, M. A. Araque Caballero, A. Baust, T. Niemczyk, E. Hoffmann, A. Marx, E. Solano, and R. Gross, Phys. Rev. Lett. 105, 100401 (2010).

[40] D. Bozyigit, C. Lang, L. Steffen, J. M. Fink, C. Eichler, M. Baur, R. Bianchetti, P. J. Leek, S. Filipp, M. P. da Silva, A. Blais, and A. Wallraff, Nat. Phys. 7, 154 (2010).

[41] C. Eichler, D. Bozyigit, C. Lang, L. Steffen, J. Fink, and A. Wallraff, Phys. Rev. Lett. 106, 220503 (2011).

[42] R. S. MacKay and S. Aubry, Nonlinearity 7, 1623 (1994).

[43] We check that the condition $\sum_{l}\left\langle a_{l}(t)\right\rangle-\left\langle a_{l}(t+\Delta t)\right\rangle \mid \leqslant 10^{-7}$ is fulfilled for the steady state. For time-periodic solutions we use the corresponding condition for the comparison of two consecutive distributions with maximal center-site amplitude.

[44] I. Pižorn, Phys. Rev. A 88, 043635 (2013).

[45] C. W. Gardiner and M. J. Collett, Phys. Rev. A 31, 3761 (1985).

[46] F. Quijandría, D. Porras, J. J. García-Ripoll, and D. Zueco, Phys. Rev. Lett. 111, 073602 (2013). 